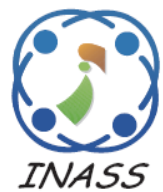

\title{
Modification of RR DBA for Performance Improvement of C-RAN on NG-PON2
}

\author{
Dwiki Kurnia $^{1 *} \quad$ Istikmal $^{1} \quad$ Ida Wahidah $^{1}$ \\ ${ }^{1}$ Telecommunication Engineering Department, \\ Faculty of Electrical Engineering, Telkom University, Indonesia \\ * Corresponding author’s Email: dwikikrnia@ student.telkomuniversity.ac.id
}

\begin{abstract}
Delay in the use of the 10 Gigabit Passive Optical Network (XG-PON) on the Centralized RAN (C-RAN) is known to be not very good since a small maximum capacity of Remote Radio Heads (RRH) used, only 8 units. Dynamic Bandwidth Allocation (DBA) is required on upstream PON to dynamically allocate bandwidth. Conventional DBA on XG-PON produces an upstream delay which is $0.6 \mathrm{~ms}$ at least. In fact, C-RAN has strict delay requirements, namely $0.3 \mathrm{~ms}$, which is why a DBA with low delay is needed as a solution. In this study, a modified Round Robin (RR) DBA algorithm using NG-PON2 is proposed with consideration of delay performance in upstream by utilizing excess bandwidth and using forecasting methods. Modified RR DBA has the best upstream delay among other DBAs that can be applied to C-RAN using a maximum of 16 Optical Network Unit (ONU) with the result of $0.261 \mathrm{~ms}$.
\end{abstract}

Keywords: Centralized RAN, NG-PON2, Dynamic bandwidth allocation, Delay.

\section{Introduction}

The emergence of the Centralized Radio Access Network (C-RAN) concept was proposed as a RAN technology capable of realizing $5 \mathrm{G}$ networks [1]. The concept of centralization in C-RAN requires a low upstream delay and a large bandwidth. Therefore, this concept requires an access network with appropriate delay and bandwidth criteria. An access network that can provide low delay, large bandwidth, high data transfer rate, and good performance is an optical access network. Next-Generation Passive Optical Network 2 (NG-PON2) is an optical access network that is currently being developed. The goal is to meet the needs of communication technology with large bandwidth. NG-PON2 can be applied to C-RAN technology to produce better delay and capacity compared to conventional PON [2]. In upstream NGPON2, Dynamic Bandwidth Allocation (DBA) is required to dynamically allocate bandwidth [3]. Conventional DBA produces a delay of at least 0.6 $\mathrm{ms}$, while C-RAN has strict delay requirements of 0.3 ms [4] so that a DBA with low delay is needed.

In the C-RAN, the Base-band Unit (BBU) is centered in one location and separated from the Radio
Unit (RU) [4-9]. Unlike the conventional RAN concept, RU and BBU are located at the same location, namely at the Base Station [5, 7, 9]. The concept of C-RAN aims for better resource management on cellular networks and simplification of RAN networks [5, 8]. In research [4], an evaluation of the Dynamic Bandwidth Allocation (DBA) on CRAN against the XG-PON access network with Time Division Multiplexing (TDM) basis was carried out which resulted in a Round Robin DBA algorithm with better throughput, delay, and latency compared to other DBA algorithms. In research [10], the application of the Demand Forecasting Dynamic Bandwidth Allocation (DF DBA) algorithm was carried out followed by a comparison with conventional DBA algorithms including RR DBA and GIANT DBA. Based on 3 analysis parameters consisting of Delay, Throughput, and Packet Delivery Ratio, DF DBA is known to get the best results among the other two. This is due to the reduction in idle time and waiting time in the DF DBA algorithm.

In this study, a modified Round Robin DBA algorithm is proposed by considering the delay performance in the upstream, namely through the use 
of excess bandwidth and the use of forecasting methods. This method can reduce idle time during upstream bandwidth allocation and avoid bandwidth overloads. In this study, we compare the performance of modified DBA with conventional RR are:

- RR DBA, that treats T-CONT in the network evenly with the number of bytes less than or equal to a predetermined fixed limit. The RR DBA algorithm uses burst time to determine the queue in the bandwidth allocation.

- Demand Forecasting DBA, that applies the forecasting method to the DBA with the aim of reducing idle time and waiting time during the transmission cycle. DF DBA implementations can provide upstream bandwidth allocation to the ONU before the ONU makes a request.

- GigaPON Access Network (GIANT) DBA is an algorithm commonly used in Giga-PON access networks. In the GIANT DBA algorithm, bandwidth allocation is differentiated by service using Transmission Container (T-CONT).

- Extended Giga PON Access Network (XGIANT) DBA is a GIANT DBA that is optimized for several parameters, including service timers, assured and non-assured ratio to traffic priority.

- Efficient Bandwidth Utilization DBA, utilizes unused bandwidth on the DBA. The available waiting line ob the byte counter on the EBU DBA can be negative where the unused remaining on the available byte counter can be used for other waiting line.

This research also considers the parameters of throughput and Packet Delivery Ratio (PDR), as well as the maximum study of Remote Radio Heads (RRH) and Optical Network Terminal (ONT) that can be aggregated using NG-PON2. Modified Round Robin DBA has the best upstream delay among other DBAs that can be applied to C-RAN which is below $0.3 \mathrm{~ms}$. The use of NG-PON2 as an access network also increases the maximum RRH and ONT capacity that can be achieved on the C-RAN system.

The rest of this paper is organized as follows: the architecture of C-RAN and NG-PON2 will be introduced in Section 2. Section 3. explains method and system model of modified DBA. Section 4. simulation and analysis results. Section 5. concludes the paper.

\section{Architecture of C-RAN and NG-PON2}

\subsection{Architecture of C-RAN}

C-RAN is a Radio Access Network that applies a centralized concept to the Baseband Unit (BBU). $\mathrm{BBU}$ functions to process information signals and

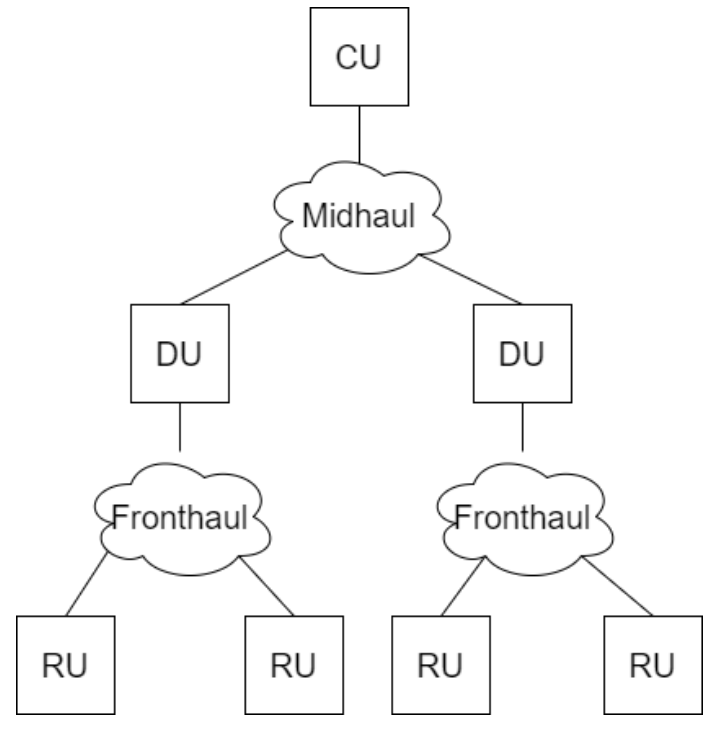

Figure. 1 C-RAN architecture model

control RRU [11, 12]. The location of the BBU device is separate from the Remote Radio Unit (RRU) device. This is different from conventional RAN which uses a distributed concept, namely Distributed RAN (D-RAN). BBU and RRU locations on D-RAN are located at the same location. Fig. 1 shows the difference graphically between C-RAN and D-RAN.

The use of C-RAN as a radio access network was found to facilitate the load balancing process. The handover process on C-RAN will also be better considering that they are in the same BBU pool. Based on infrastructure, the C-RAN concept can save power consumption when compared to D-RAN because C-RAN does not require the implementation of an Indoor Site on the End Site or RRU side [13]. Furthermore, C-RAN has strict delay requirements, a maximum of $0.3 \mathrm{~ms}$ [4] which therefore requires a DBA which results in low delay for C-RAN to operate properly.

\subsection{Architecture of NG-PON2}

In 2010, the Full-Service Access Network (FSAN) Group initiated and determined the standard used for NG-PON2 technology. The discussion at the FSAN forum is about the latest telecommunications technology, especially PON technology. The ITU-T recommendation regarding NG-PON2 has been set in the G.989 series which includes G.989, G.989.1, G.989.2. The general architecture of NG-PON2 is shown in Fig. 2.

Several recommendations and requirements that are the main targets for NG-PON2 technology include the following $[14,15]$ : 


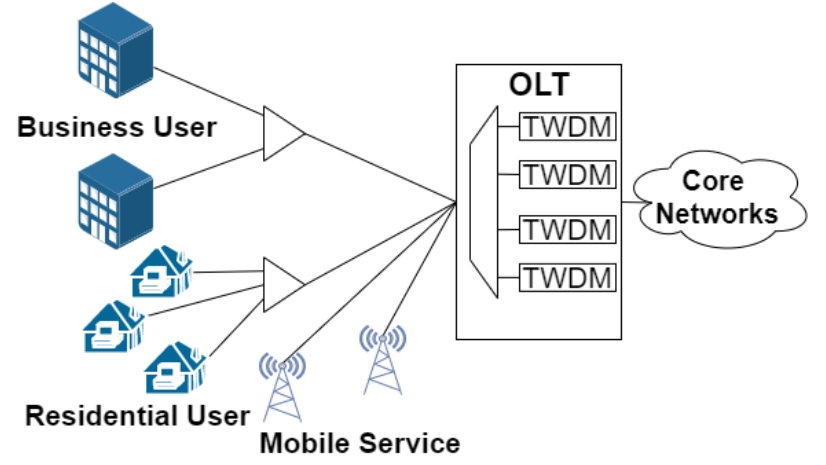

Figure. 2 NG-PON2 architecture model

- The aggregation capacity of each OLT with a bit rate of $40 \mathrm{Gbit} / \mathrm{s}$ downstream and $10 \mathrm{Gbit} / \mathrm{s}$ upstream.

- Supports more than 64 ONUs or subscribers per one OLT.

- Compatible with previous PON technologies.

\section{Method and system model}

The design of the C-RAN system model using the NG-PON2 access network is carried out through simulation using NS3 software. The main part of the network system is the transmitter part in the form of an Optical Line Terminal (OLT), the transmission medium, and Optical Network Unit (ONU) as optical receiver.

The design model of the C-RAN system using the NG-PON2 access network is illustrated in Fig. 3 The design of this system uses 4 OLT consisting of a transmitter and a receiver. The OLT device is placed in the Central Office which consists of a Laser Transmitter, Photodetector Receiver, Modulator, and Multiplexer [16]. The transmission block, namely the Optical Distribution Network (ODN), consists of a G.652c optical cable and 3 splitters with different total splitter ratios. On the receiver side, there is an ONU terminal which consists of a photodetector and a filter to process signals from the OLT. The ONU port is distributed on the C-RAN through the Baseband Unit (BBU) and connected to the Remote Radio Unit (RRU) [17].

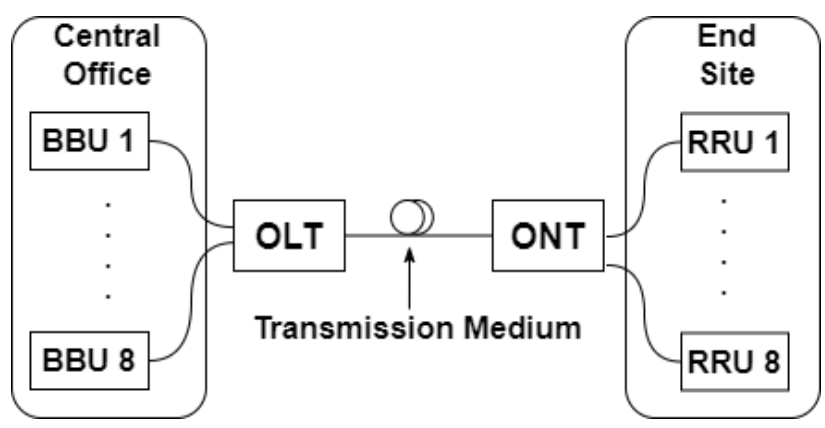

Figure. 3 C-RAN system model on NG-PON2
Table 1. Transmitter parameter

\begin{tabular}{|c|c|}
\hline Parameter & Value \\
\hline Upstream bitrate per channel & $2.5 \mathrm{Gbps}$ \\
\hline OLT aggregation & 4 OLT \\
\hline
\end{tabular}

\subsection{Transmitter parameters}

The optical transmitter is the main part whose function is to send information from the center to the end-user with the name of the OLT device. Table 1 presents the specifications of the transmitter used based on the ITU-T G.989.2 recommendation [18].

On the transmitter side, 4 OLT aggregation is used with an upstream bit rate and a bitrate per channel of $2.5 \mathrm{Gbps}$ which results in a total bitrate aggregation of $10 \mathrm{Gbps}$.

\subsection{Transmission media parameters}

Optical Distribution Network (ODN) is a liaison between the center to the end-user in the optical fiber communication system. In this study, the splitter ratios used were 1:2, 1:4, 1:8, 1:16, 1:32, 1:64, and $1: 128$.

This study also used a splitter at 2 points. The first splitter is placed on the ODC with a ratio of 1:2, 1:4, and 1:8, while the second splitter is placed on the ODP side with a ratio of $1: 8$ and $1: 16$ with the addition of one splitter feeder on the central site of the OLT. The propagation delay used in this case is $0.1 \mathrm{~ms}$ assuming the transmission distance is $10 \mathrm{~km}$.

\subsection{Receiver parameters}

The light will be received by the Receiver after being transmitted through the ODN by first passing through the photodetector and optical filter. The function of the photodetector is to detect light and convert it into an electric current [19]. After the light is converted into an electric current, the current will pass through a Low Pass Filter (LPF) where unwanted signals will be removed [16]. The maximum transmission distance that can be achieved on NG-PON2 is $40 \mathrm{~km}[14,15]$.

\subsection{Parameters of C-RAN}

The components of C-RAN consist of BBU and RRU. The two components are placed in different locations. BBU is located at the Central Office, while RRU is located at the End Site. The minimum upstream delay that can operate on the C-RAN fronthaul is $0.3 \mathrm{~ms}$. The average RRU connected to each ONT is assumed to be 3 RRU according to the number of sectoral antennas. Table 2 provides 
Table 2. C-RAN parameter

\begin{tabular}{|c|c|}
\hline Parameter & Value \\
\hline Simulation time & $10 \mathrm{~s}$ \\
\hline Number of RRH / ONU & $2,4,8,16,32,64,128$ \\
\hline Propagation delay & $0.1 \mathrm{~ms}$ \\
\hline
\end{tabular}

information on the C-RAN parameters used in this study.

\subsection{Modified DBA algorithm}

The algorithm starts by determining the variables as presented in Fig. 4. The next step is to select a buffer index on T-CONT to perform an occupancy report and determine the bandwidth allocation for TCONT. If the DBA cycle reaches 100 times, then bandwidth forecasting is carried out followed by determining the bandwidth allocation on the TCONT. However, if the DBA cycle does not reach 100 times, the bandwidth allocation is determined without performing a bandwidth forecasting process. The number of transmission cycles is determined based on the number of references [10] with better DBA results when compared to RR DBA and GIANT DBA. If the bandwidth in the occupancy report is greater than the maximum bandwidth allocation, then the bandwidth allocation on the j-th index, namely $W^{j}$ is the maximum bandwidth allocation of Wmax plus the excess bandwidth of $E x^{j}$. This stage is followed by filtering by adjusting parameter criteria such as delay, throughput, and packet delivery ratio. If the cycle meets the parameter criteria, then the calculation of excess bandwidth for the allocation of T-CONT in the next cycle needs to be done. Variables need to be re-determined if they do not meet the criteria.

This study also used a splitter at 2 points. The first splitter is placed on the ODC with a ratio of 1:2, 1:4, and $1: 8$, while the second splitter is placed on the ODP side with a ratio of $1: 8$ and $1: 16$ with the addition of one splitter feeder on the central site of the OLT. The propagation delay used in this case is $0.1 \mathrm{~ms}$ assuming the transmission distance is $10 \mathrm{~km}$.

In DBA, the transmission cycle time $T_{\text {cycle }}$ is determined by the data to send time $T_{D S}$, idle time $T_{\text {idle }}$, OLT process time $T_{O L T}$, and time to send the bandwidth allocation from OLT to ONU or $T_{\text {grant }}$ which is expressed by Eqs (1) and (2) [10].

$$
\begin{gathered}
T_{\text {waiting }}=T_{\text {OLT }}+T_{\text {grant }} \\
T_{\text {cycle }}=T_{D S}+T_{\text {idle }}+T_{\text {waiting }}
\end{gathered}
$$

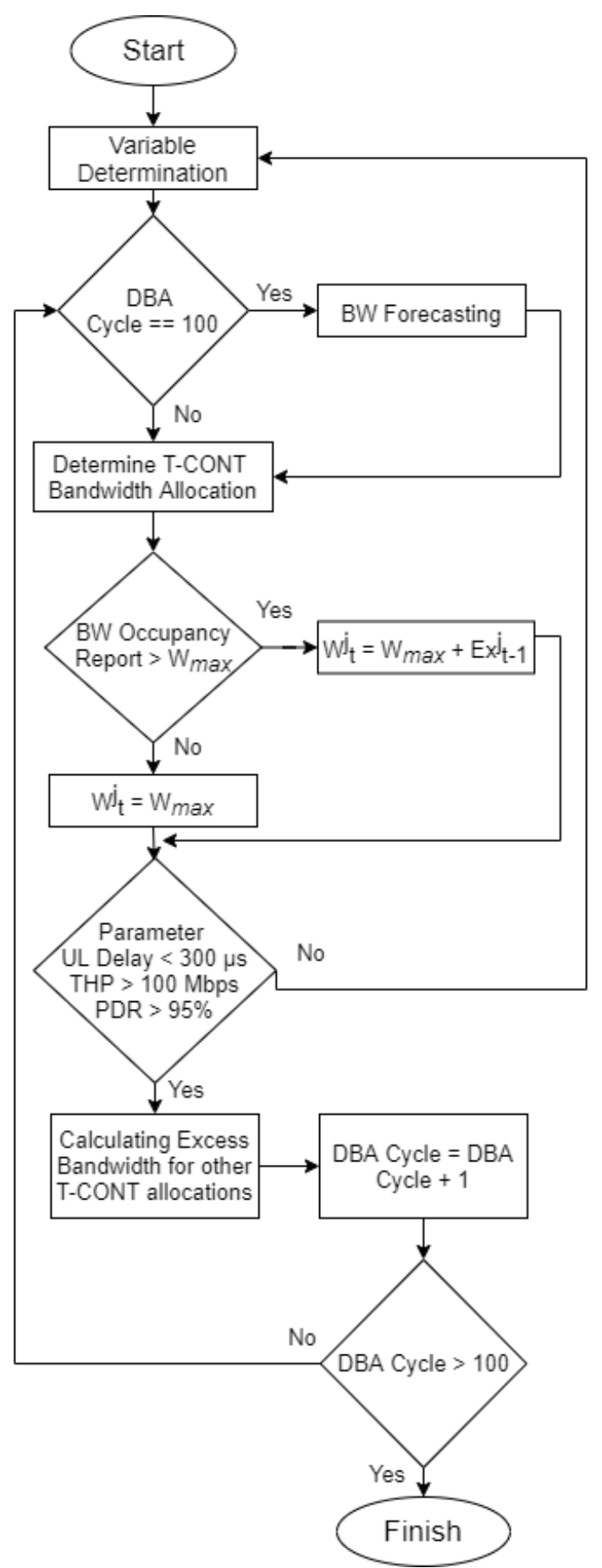

Figure. 4 Modification algorithm flowchart

The use of bandwidth forecasting in determining bandwidth allocation can reduce idle time so that the transmission cycle time becomes faster. Allocation process bandwidth using bandwidth forecasting on the modified RR DBA is visualized in Fig. 5. The bandwidth forecasting method will allocate bandwidth before the ONU makes a bandwidth request to the OLT. In a conventional DBA, bandwidth allocation can be processed after the ONU has made a bandwidth request to the OLT. 


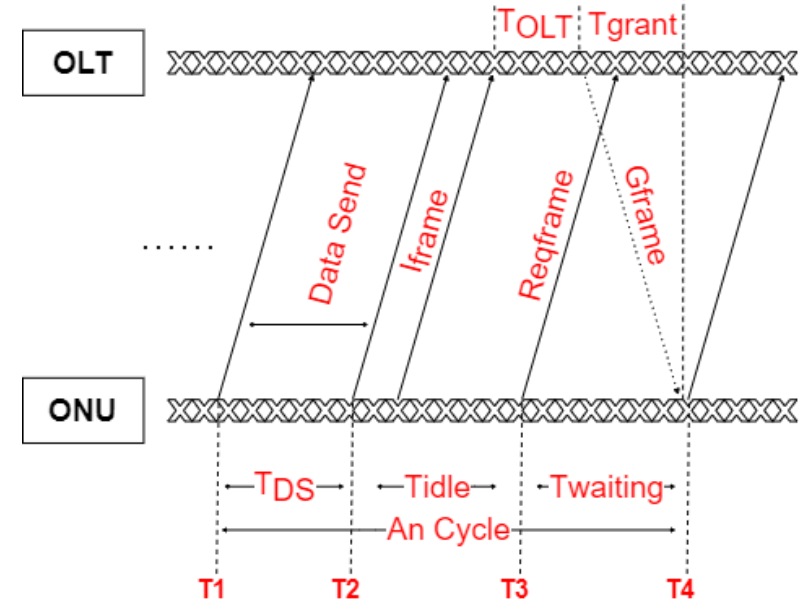

Figure. 5 Allocation process of modified DBA

At each upstream frame, the ONU sends some waiting data and a report from the Dynamic Bandwidth Report (DBRu) which contains the amount of data during the waiting time to the OLT. $R e q[i]$ is the request for DBRu in structure $i$ and Data[i] is the quantity of data transmitted in frame $i$ so that the arrival rate data in the T-CONT waiting time can be calculated, namely by the Eq. (3) [10]

$$
\lambda_{T C O N T}=\frac{\operatorname{Req}[i]+\operatorname{Data}[i]-\operatorname{Req}[i-1]}{\Delta T}
$$

The concept of statistical modeling is used to predict the size of demand. OLT uses a circular buffer to store the last 100 Data[i] and Req[i] values. This value can be used to calculate the arrival rate data for each T-CONT. In each cycle, the mean and standard deviation of $\lambda_{T C O N T}$ or arrival rate data are calculated using the circular buffer and used to forecast the size of $L_{T C O N T}$ demand using a normal distribution [10].

$$
L_{T C O N T}=(N(\mu, \sigma) \times \Delta T)+128
$$

The next step accepts the $L_{T C O N T}$ as the amount of data that arrives at the ONU T-CONT waiting for the last frame duration of $\Delta T$ and is used to calculate bandwidth allocation to T-CONT on the next BW map. Next, the constant 128 is added to accommodate frames in the DBRu report and error estimation.

\section{Analysis and discussion}

In this simulation, the upstream bandwidth used is $10 \mathrm{Gbps}$. The propagation delay is set to $1 \mathrm{~ms}$ according to the ITU-T standard, with an approach distance of $10 \mathrm{~km}$. The maximum data transfer rate on the upstream link is $80 \%$ of the total maximum bandwidth capacity of NG-PON2 for network protection. DBA comparison is done by comparing 6 DBAs, namely the proposed DBA or Optimized
DBA [4], Demand Forecasting DBA [10], conventional DBA algorithms including Round Robin DBA, EBU DBA, GIANT DBA, and XGIANT DBA. Following are several scenarios used in this study.

- In scenario 1 , the ONU is set to vary, from 2 ONU, 4 ONU, 8 ONU, 16 ONU, 32 ONU, 64 $\mathrm{ONU}$, and $128 \mathrm{ONU}$ with a fixed distance of 10 $\mathrm{km}$ where the traffic load is set at $50 \%$.

- In scenario 2, the distance is set to vary, ranging from $10 \mathrm{~km}, 20 \mathrm{~km}, 30 \mathrm{~km}$, and $40 \mathrm{~km}$ with a fixed number of 16 ONUs where the traffic load is set at $50 \%$.

- In scenario 3, the traffic load is set to vary, including $10 \%, 30 \%, 50 \%, 70 \%$, and $90 \%$ with a fixed number of ONUs is 16 ONUs and the distance from OLT to ONT is $10 \mathrm{~km}$.

\subsection{Upstream delay analysis}

In scenario 1 , the number of ONUs used varies from 2 ONUs to 128 ONUs with a distance of $10 \mathrm{~km}$ and a traffic load of $50 \%$. The graph of the simulation results of the upstream delay parameter in Scenario 1 is presented in Fig. 6.

Based on the graph in Fig. 6, upstream delay on 2 to $16 \mathrm{ONU}$ produces the same value except for EBU DBA. The significant difference after $16 \mathrm{ONU}$ with the shortest upstream delay value is Modified DBA of $0.261666 \mathrm{~ms}$ where the longest is EBU DBA of $0.426849 \mathrm{~ms}$. In terms of performance, the smaller the upstream delay, the better the performance. DBA modifications on the upstream delay parameter, therefore, get the best results among other DBAs. This is due to the reduction in idle time and waiting time in the modified DBA algorithm due to the use of forecasting and idle bandwidth in bandwidth allocation.

Based on the delay, the modified DBA can be used on C-RAN up to $16 \mathrm{ONU}$ with the result of delay below $0.3 \mathrm{~ms}$, which is $0.261666 \mathrm{~ms}$. XGIANT DBA, EBU DBA, and RR DBA produce delays below $0.3 \mathrm{~ms}$ on 8 ONUs.

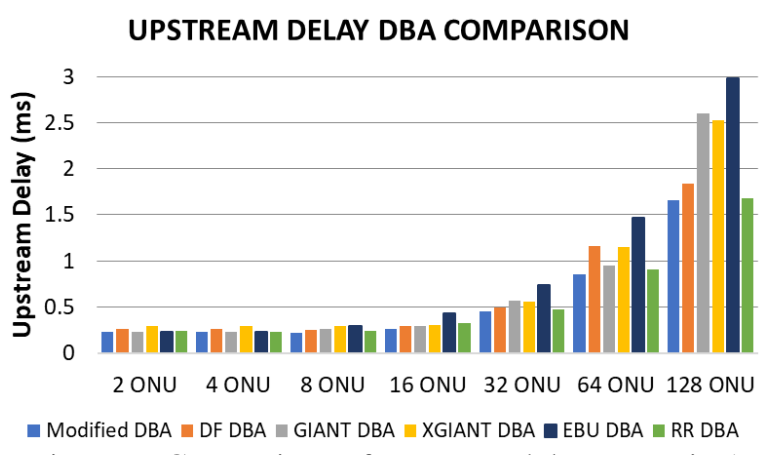

Figure. 6 Comparison of upstream delay scenario 1 


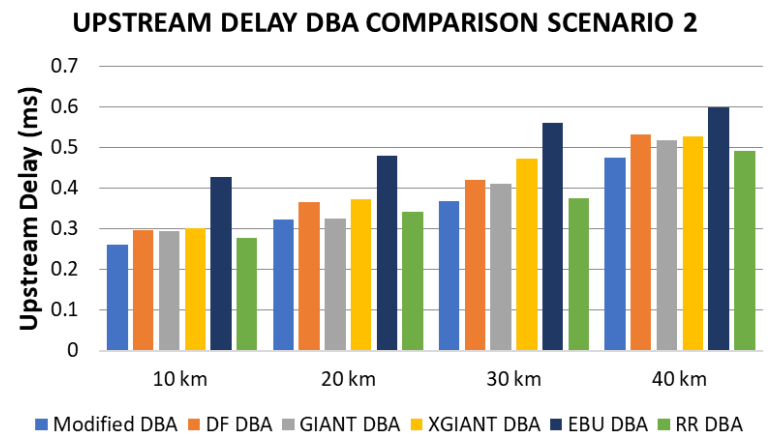

Figure. 7 Comparison of upstream delay scenario 2

This allows DBA Modifications to be flexibly applied to a varying number of ONUs, even up to 16 ONUs. The effect of the number of ONUs on the upstream delay parameter, in this case, is directly proportional as the more number of ONUs used, the higher the upstream delay generated. The reason is that the queue process for bandwidth allocation on each ONU increases as the number of ONUs served increases.

In scenario 2, the distance from OLT to ONU is set to vary, ranging from $10 \mathrm{~km}, 20 \mathrm{~km}, 30 \mathrm{~km}$, and $40 \mathrm{~km}$ using 16 ONUs and $50 \%$ traffic load. The graph of upstream delay simulation results in Scenario 2 can be seen in the following figure.

Based on the graph in Fig. 7, upstream delay at distance $10 \mathrm{~km}$ using Modified DBA, DF DBA, GIANT DBA, XGIANT DBA, EBU DBA, and RR DBA respectively are $0.261666 \mathrm{~ms}, 0.295494 \mathrm{~ms}$, $0.293558 \mathrm{~ms}, 0.301499 \mathrm{~ms}, 0.426849 \mathrm{~ms}$, and $0.277593 \mathrm{~ms}$. Upstream delay performance at a distance of $20 \mathrm{~km}, 30 \mathrm{~km}$, and $40 \mathrm{~km}$ provides a value linearly and directly proportional to the resulting upstream delay. This can occur due to an increase in propagation delay as the distance changes further.

Based on the results of the delay performance in Fig. 7, Modified DBA provides the lowest upstream delay value compared to other DBAs, while the highest upstream delay is obtained when using EBU DBA. The results of upstream delay in scenario 2 give the same conclusion as scenario 1, where Modified DBA is the best compared to other DBAs. Based on the delay, all DBAs can be used on C-RAN up to a distance of $10 \mathrm{~km}$ with a delayed result of less than $0.3 \mathrm{~ms}$.

In scenario 3 , the traffic load is varied, starting from $10 \%, 30 \%, 50 \%, 70 \%$, and $90 \%$ with a distance of $10 \mathrm{~km}$ and $16 \mathrm{ONU}$. The graph of upstream delay simulation results in Scenario 3 can be seen in the following figure. Based on the graph in Fig. 8, the difference between 50\% traffic load and 90\% traffic load is $14.81 \mu \mathrm{s}$. Based on the average difference, it can be seen that the use of varying traffic loads does not directly affect the upstream delay. Upstream

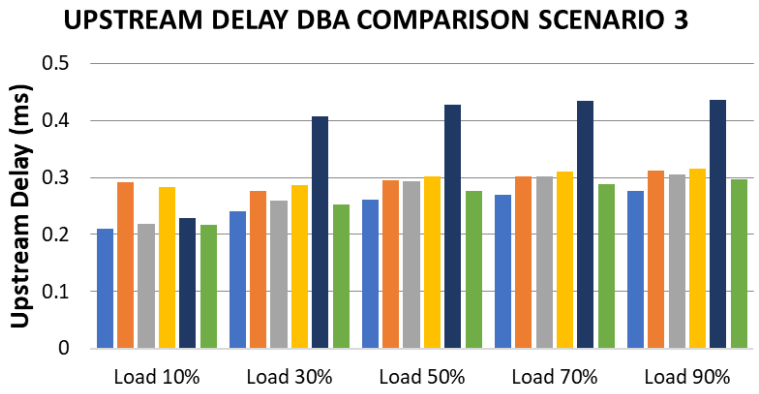

$\square$ Modified DBA $\square$ DF DBA $\square$ GIANT DBA $₫$ XGIANT DBA $\llbracket$ EBU DBA $\square$ RR DBA Figure. 8 Comparison of upstream delay scenario 3

delay generated at 70\% traffic load on Modified DBA, DF DBA, GIANT DBA, XGIANT DBA, EBU DBA, and RR DBA are $0.268731 \mathrm{~ms}, 0.301054 \mathrm{~ms}$, $0.301868 \mathrm{~ms}, 0.310058 \mathrm{~ms}, 0.434443 \mathrm{~ms}$, and $0.28874 \mathrm{~ms}$, respectively.

Based on the results of the upstream delay performance, Modified DBA gets the shortest upstream delay while EBU DBA has the longest upstream delay. This is following the results obtained in scenario 1 and scenario 2 so that it can be concluded from the three scenarios, Modification of DBA produces the shortest upstream delay when compared to the other five scenarios. Reducing idle time and waiting time and using excess bandwidth in the Modified DBA algorithm is the main contribution in producing a better upstream delay compared to other DBAs.

\subsection{Throughput analysis}

Based on the graph in Fig. 9, the throughput value obtained by using 2 ONUs is not much different, except for XGIANT DBA. The resulting throughput is throughput per ONU. The throughput values have significant differences in 16 ONUs obtained from Modified DBA, DF DBA, GIANT DBA, XGIANT DBA, EBU DBA, and RR DBA, respectively are 134,099 Mbps, 133,269 Mbps, 133,216 Mbps, 132,513 Mbps, 93.0894 Mbps, and 134.095 Mbps. Based on this value, Modified DBA has the highest throughput value with a difference that is not much different from DF DBA and RR DBA. Meanwhile,

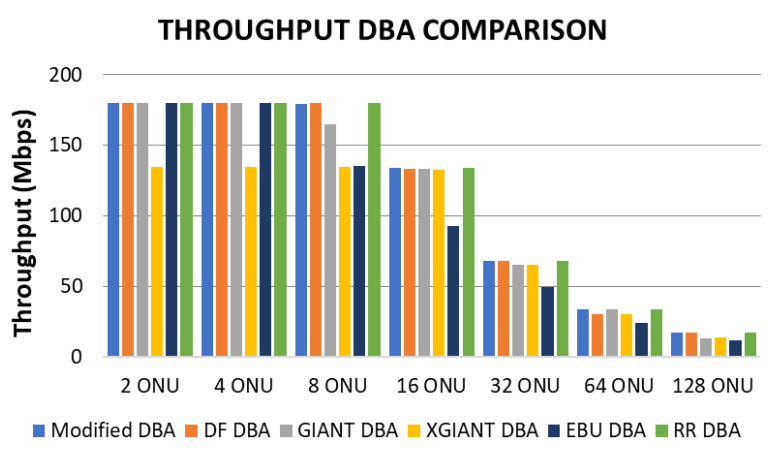

Figure. 9 Comparison of throughput scenario 1 
THROUGHPUT DBA COMPARISON SCENARIO 2

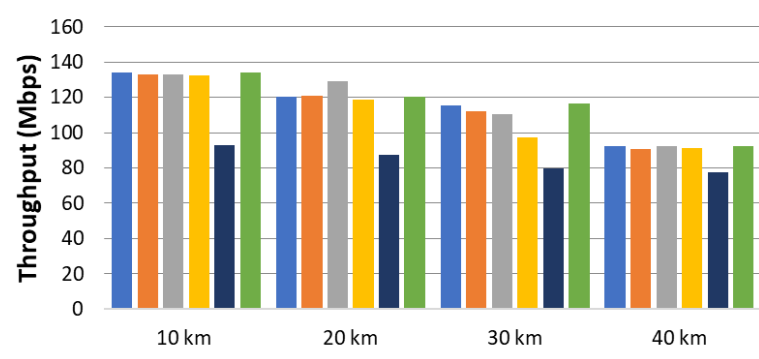

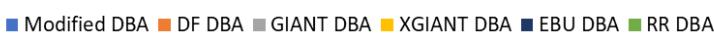

Figure. 10 Comparison of throughput scenario 2

the effect of reducing idle time on the DBA modification on the resulting throughput does not affect the throughput performance results.

Theoretically, LTE with a bandwidth of $20 \mathrm{MHz}$ requires a throughput of $100 \mathrm{Mbps}$ [20]. Thus, the achievable uplink throughput exceeds $100 \mathrm{Mbps}$ maximum using 16 ONUs on all DBAs, except EBU DBAs. It can be concluded that the comparison between throughput and the number of ONUs based on those presented in Fig. 9 is the opposite. That is, the more the number of ONU, the resulting throughput will be smaller. The decrease in throughput on the number of ONUs is due to the distribution of the total bandwidth following the number of ONUs served.

Throughput in Scenario 2 using EBU DBA has a significant difference compared to other DBAs. In Fig. 10, the throughput between the other five DBAs has not many different results. At a distance of $30 \mathrm{~km}$, throughput using Modified DBA, DF DBA, GIANT DBA, XGIANT DBA, EBU DBA, and RR DBA are 115,221 Mbps, 111,924 Mbps, 110,241 Mbps, 97.3461 Mbps, 79.7086 Mbps, and 116,276 Mbps, respectively. RR DBA was found to have the highest throughput when compared to other DBAs, with a difference that was not much different from the Modified DBA.

The throughput value is inversely proportional to the distance, where the higher the distance, the lower the resulting throughput. Increasing the distance results in a propagation delay that is directly

THROUGHPUT DBA COMPARISON SCENARIO 3

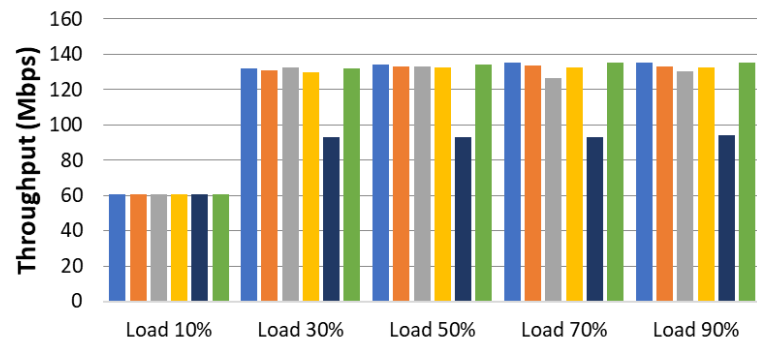

- Modified DBA $\llbracket$ DF DBA $\llbracket$ GIANT DBA $\llbracket$ XGIANT DBA $\llbracket$ EBU DBA $\llbracket$ RR DBA Figure. 11 Comparison of throughput scenario 3 proportional so that it can be concluded that the propagation delay has a direct effect on the resulting throughput parameters.

In Scenario 3 presented in Fig. 11, the traffic load is set to vary to produce a directly proportional throughput. The higher the regulated traffic load, the higher the throughput generated. At 70\% traffic load, the throughput generated in Modified DBA, DF DBA, GIANT DBA, XGIANT DBA, and RR DBA respectively is $135,404 \mathrm{Mbps}, 133,476 \mathrm{Mbps}$, 126.587 Mbps, 132.554 Mbps, 93.2597 Mbps, and 135,406 Mbps. Modified DBA and RR DBA produce the highest throughput when compared to other DBAs. The increase in throughput on traffic loads is experienced in each DBA but does not apply to EBU DBA. This is because EBU DBA using a bandwidth efficiency method that increases throughput when the traffic load is getting smaller.

\subsection{Packet delivery ratio analysis}

In Scenario 1 shown in Fig. 12, 4 ONU Modified DBA, DF DBA, GIANT DBA, XGIANT DBA, and

RR DBAs have PDR values of $89.78 \%, 89.77 \%$, $89.78 \%, 86.33 \%, 89.78 \%$, and $89.78 \%$. Meanwhile, the PDR values using 8 ONUs were $95.50 \%, 95.50 \%$, $94.96 \%, 93.52 \%, 92.21 \%$, and $95.51 \%$. Modified DBA and RR DBA have the highest PDR value when compared to other DBAs. The lowest PDR value was found in 2 ONUs with an average PDR of $79.27 \%$, below the $95 \%$ threshold. The increase in the number of ONUs is directly proportional to the resulting PDR. In $2 \mathrm{ONU}$, the Bitrate owned is the highest compared to the number of other ONU because the total Bitrate of NG-PON2 is allocated based on the number of ONUs, increasing PDR performance.

Based on Fig. 13, it can be seen that the effect of distance on the resulting PDR is inversely proportional, where the greater the distance produced, the lower the PDR obtained for each DBA. At a distance of $40 \mathrm{~km}$, the PDR generated by Modified DBA, DF DBA, GIANT DBA, XGIANT DBA, and RR DBA were 95.51\%, 95.51\%, 95.67\%, 96.20\%,

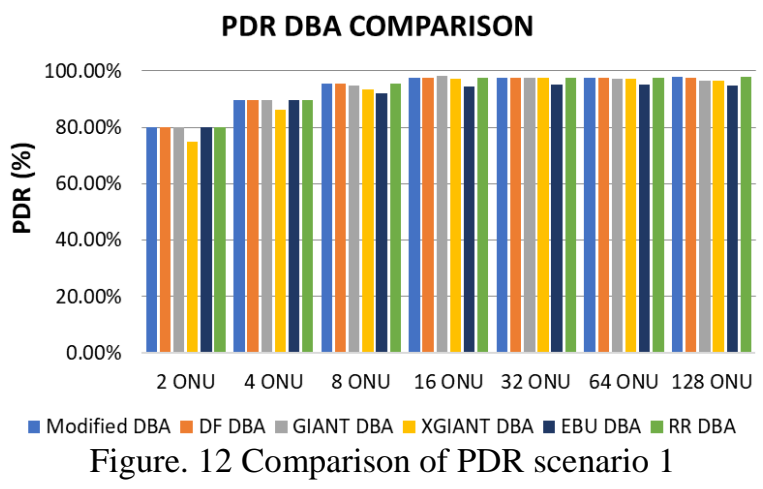


PDR DBA COMPARISON SCENARIO 2

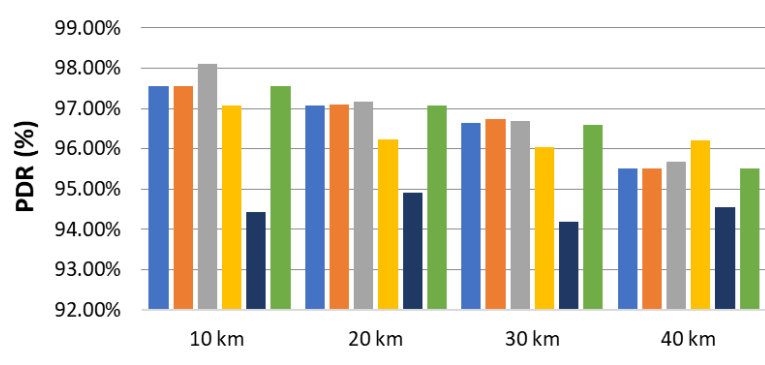

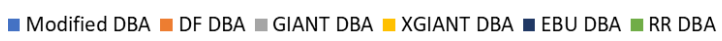
Figure. 13 Comparison of PDR scenario 2

\section{PDR DBA COMPARISON SCENARIO 3}

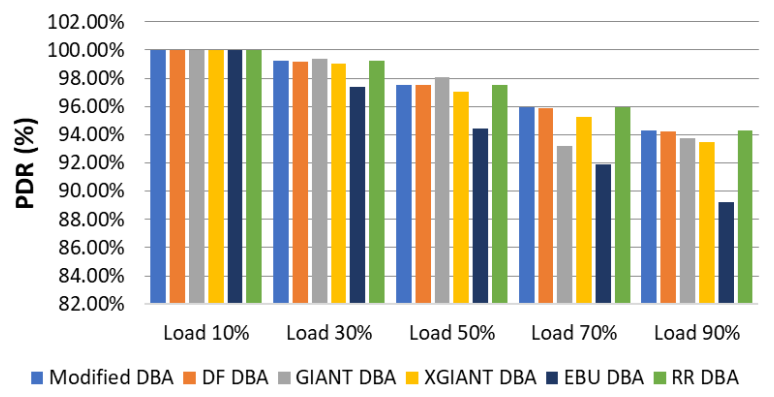

Figure. 14 Comparison of PDR scenario 3

$94.56 \%$ and $95.51 \%$, respectively. The average PDR produced is $95.49 \%$ where the highest PDR is $95.51 \%$, while the lowest is $94.56 \%$. The results of PDR at a distance of $40 \mathrm{Km}$ get values above $95 \%$, except for EBU DBA, so that EBU DBA does not meet the PDR parameter threshold. Based on Fig. 13, it can be concluded that the distance and propagation delay can affect the resulting PDR.

At $70 \%$ traffic load, PDR with Modified DBA, DF DBA, GIANT DBA, XGIANT DBA, EBU DBA, and RR DBA were 95.93\%, 95.88\%, 93.19\%, $95.24 \%, 91.87 \%$, and $95.93 \%$. The highest PDR was found in Modified DBA and RR DBA, while the lowest PDR was in EBU DBA, 91.87\%.

Based on Fig. 14, it can be seen that the results of the PDR on the traffic load are inversely proportional. That is, the greater the traffic load, the lower the PDR generated. Fig. 14 shows that the results of the PDR on the traffic load are inversely proportional where the greater the traffic load, the lower the PDR generated.

\section{Conclusion}

Based on the analysis of upstream delay parameters in scenario 1, scenario 2, and scenario 3, it was found that Modified DBA has better parameter results when compared to other DBAs. Parameter results sequentially followed by RR DBA, DF DBA, GIANT DBA, XGIANT DBA, and EBU DBA. Based on throughput and PDR parameters, Modified
DBA, RR DBA, and DF DBA have values that are not much different.

In scenario 1 , the number of ONUs that can be used in the Modified DBA is 16 ONUs based on the parameters of upstream delay, throughput, and PDR. Referring to scenario 2, the maximum distance that can be achieved is $10 \mathrm{~km}$. Traffic loads that vary from $10 \%, 30 \%, 50 \%, 70 \%$, and $90 \%$ can be achieved with the results of parameters that applied to C-RAN. In the future work, the modified DBA can use a more advanced forecasting scheme and have more considerations for high-level accuracy.

\section{Conflicts of Interest}

The authors declare no conflict of interest.

\section{Author Contributions}

D. Kurnia: Conceptualization, modification, software simulation, resources, investigation, visualization, writing original draft preparation and editing. Istikmal: Validation, supervision, funding acquisition and formal analysis. I. Wahidah: Supervision, project administration, validation and data curation.

\section{References}

[1] A. M. Alba, S. Janardhanan, and W. Kellerer, "Enabling Dynamically Centralized RAN Architectures in 5G and Beyond", IEEE Transactions on Network and Service Management, 2021.

[2] A. Zaouga, A. Sousade, M. Najja, and P. Monteiro, "Low Latency Dynamic Bandwidth Allocation Algorithms for NG-PON2 to support 5G Fronthaul and Data Services", In: Proc. of International Conference on Transparent Optical Networks, pp. 1-4, 2019.

[3] ITU-T G.984.3, "Gigabit-capable passive optical networks (G-PON): Transmission convergence layer specification", 2014.

[4] A. M. Mikaeil, W. Hu, T. Ye, and S. B. Hussain, "Performance Evaluation of XG- PON Based Mobile Front-Haul Transport in Cloud-RAN Architecture", IEEE/OSA Journal of Optical Communications and Networking, Vol. 9, No. 11, pp. 984-994, 2017.

[5] Y. Nakayama, D. Hisano, T. Tsutsumi, and K. Maruta, "Novel C-RAN Architecture with PON based Midhaul and Wireless Relay Fronthaul", In: Proc. of IEEE 17th Annual Consumer Communications \& Networking Conference, pp. $1-6,2020$. 
[6] M. Fiorani, A. Rostami, L. Wosinska, and P. Monti, "Transport Abstraction Models for an SDN-Controlled Centralized RAN", IEEE Communications Letters, Vol. 19, No. 8, pp. 1406-1409, 2015.

[7] S. Gulati, B. Natarajan, S. Kalyanasundaram, and R. Agrawal, "Performance Analysis of Centralized RAN Deployment with Non-Ideal Fronthaul in LTE-Advanced Networks", In: Proc. of IEEE 83rd Vehicular Technology Conference, pp. 1-5, 2016.

[8] B. Mahapatra, A. K. Turuk, S. K. Patra, and R. Kumar, "Optimal Placement of Centralized BBU (C-BBU) for Fronthaul and Backhaul Optimization in Cloud-RAN Network", In: Proc. of International Conference on Information Technology, pp. 107-112, 2017.

[9] R. S. Oliveira, C. R. L. Francês, J. C. W. A. Costa, D. F. R. Viana, M. Lima, and A. Teixeira, "Analysis of the Cost-Effective Digital Radio over Fiber System in the NG-PON2 context", In: Proc. of 16th International Telecommunications Network Strategy and Planning Symposium, pp. $1-6,2014$.

[10] K. A. Memon, K. H. Mohammadani, N. U. Ain, A. Shaikh, S. Ullah, Q. Zhang, B. Das, R. Ullah, F. Tian, and X. Xin, "Demand Forecasting DBA Algorithm for Reducing Packet Delay with Efficient Bandwidth Allocation in XG-PON", Electronics Journal, Vol. 8, pp. 147, 2019.

[11] H. Rastegarfar, T. Svensson, and N. Peyghambarian, "Optical Layer Routing Influence on Software-Defined C-RAN Survivability", IEEE/OSA Journal of Optical Communications and Networking, Vol. 10, No. 11, pp. 866-877, 2018.

[12] B. Mahapatra, R. Kumar, A. K. Turuk, S. K. Patra, "A Multilevel Co-operative Load Balancing Algorithm for C-RAN Architecture", Journal of Digital Communication Networks, Vol. 5, pp. 308-316, 2019.

[13] A. Boaventura, "Base Station Virtualization Advantages and Challenges", LTE Latin America Forum, 2015.

[14] M. Carroll, D. Nesset, and P. Dawes, "FSAN Highlights and NG-PON2 Standards Update", Full-Service Access Network, 2015.

[15] P. Kourtessis, W. Lim, N. Merayo, Y. Yang, and J. M. Senior, "Efficient T-CONT-Agnostic Bandwidth and Wavelength Allocation for NGPON2", IEEE/OSA Journal of Optical Communications and Networking, Vol. 11, No. 7, 383-396, 2019.

[16] G. Keiser, "Optical Fiber Communication Third Edition, McGraw-Hill Higher Education”, 2010.
[17] ITU-T G.Sup.66, "5G Wireless Fronthaul Requirements in a Passive Optical Network Context", 2018.

[18] ITU-T G.989.2, “40-Gigabit-capable passive optical networks 2 (NG-PON2): Physical media dependent (PMD) layer specification”, 2014.

[19] N. Blaunstein, S. Engelberg, E. Krouk, and M. Sergeev, "Optical Sources and Detectors", Fiber Optic and Atmospheric Optical Communication, pp.117-131, 2020.

[20] Z. Tayq, "Fronthaul integration and monitoring in 5G networks", Signal and Image Processing HAL Archives-Overtes, 2018. 